\title{
The Non-Conserved C-Terminal Segments of Sine Oculis Homeobox (SIX) Proteins Confer Functional Specificity
}

\author{
Brandon P. Weasner and Justin P. Kumar ${ }^{\star}$ \\ Department of Biology, Indiana University, Bloomington, Indiana
}

\begin{abstract}
Summary: The Sine Oculis Homeobox (SIX) proteins play critical roles in organogenesis and are defined by the presence of two evolutionarily conserved functional motifs: a homeobox DNA binding domain and the SIX protein-protein interaction domain. Members of this transcription factor family can be divided into three subgroups: Six 1/2, Six $4 / 5$, and Six 3/6. This partitioning is based mainly on protein sequence similarity and genomic architecture, and not on specificities of DNA binding or binding partners. In fact, it is well demonstrated that members of the different subgroups can bind to and activate common transcriptional targets as well as form biochemical complexes with communal binding partners. Here we report that the $\mathrm{C}$-terminal segment, which is not conserved across different SIX subfamilies, may serve to functionally distinguish individual SIX proteins. In particular, we have dissected the C-terminal region of Optix, the Drosophila ortholog of mammalian Six $3 / 6$, and identified three regions that distinguish Optix from Sine Oculis, the fly homolog of Six 1/2. Two of these regions have been preserved in all Six $3 / 6$ family members while the third section is present only within Optix proteins in the Drosophilids. The activities of these regions are required, in unison, for Optix function. We suggest that biochemical/functional differences between members of large protein families as well as proteins encoded by duplicate genes can, in part, be attributed to the activities of nonconserved segments. Finally, we demonstrate that a subset of vertebrate SIX proteins has retained the ability to function during normal fly eye development but have lost the ability to induce the formation of ectopic eyes.
\end{abstract}

\section{Keywords}

sine oculis; optix; Six3; Six6; retinal determination; eye specification

\section{INTRODUCTION}

The development of the Drosophila compound eye begins in embryogenesis when a small set of cells within the dorso-lateral regions of the head begin to express the Pax6 genes eyeless (ey) and twin of eyeless (toy; Czerny et al., 1999; Quiring et al., 1994). These cells self organize to form a monolayer epithelial sheet called the eye-antennal imaginal disc (reviewed in Cohen, 1993; Held, 2002). As development proceeds, the remaining members of the retinal determination network (RD) including sine oculis (so), optix, eyes absent (eya), dachshund (dac), teashirt (tsh), and homothorax (hth) are expressed within the eye field (Bessa et al., 2002; Bonini et al., 1993; Cheyette et al., 1994; Mardon et al., 1994; Serikaku and O'Tousa, 1994; Pai et al., 1998; Pan and Rubin, 1998; Seimiya and Gehring, 2000; Singh et al., 2002). The simultaneous expression of all RD genes is sufficient to commit the eye primordium to adopting a retinal fate (Kumar and Moses, 2001). At the beginning of the third and final instar,

(C) 2009 Wiley-Liss, Inc.

*Correspondence to: Justin P. Kumar, Department of Biology, Indiana University, Bloomington, IN 47405. E-mail: jkumar@ indiana.edu. 
pattern formation begins in the retina when a wave of morphogenesis is initiated at the posterior margin of the eye field. This morphogenetic front proceeds towards the anterior edge and its movement can be monitored by a dorso-ventral indentation in the epithelium called the morphogenetic furrow (Ready et al., 1976). The passage of the furrow across the eye primordium transforms a vast array of undifferentiated and nonpatterned cells into a repetitive array of unit eyes or ommatidia (Ready et al., 1976; Wolff and Ready, 1991).

so, optix, and DSix4 encode Sine Oculis Homeobox (SIX) transcription factors. Of these genes, so and optix are expressed in the developing fly retina (Cheyette et al., 1994; Seimiya and Gehring, 2000; Serikaku and O'Tousa, 1994) while DSix4 expression is restricted to the gonad and several mesodermal derivatives (Clark et al., 2006; Kirby et al., 2001). Within the anterior regions of the eye field So is distributed in a broad swathe of cells adjacent to the morphogenetic furrow and in cells that will give rise to the ocelli. Posterior to the advancing furrow, So is present in all cells including developing photoreceptor neurons and undifferentiated cells (Cheyette et al., 1994; Serikaku and O'Tousa, 1994). Optix protein, however, is restricted to cells anterior to the furrow and completely overlaps with So distribution in this area (Seimiya and Gehring, 2000) except for the ocellar regions in which optix is expressed just anterior to the medial ocellus (Salzer and Kumar, unpublished data).

Loss-of-function mutants indicate that both so and optix play important roles in eye development. Flies in which so expression has been completely eliminated (through mutations in eye specific enhancers) completely lack compound eyes (Cheyette et al., 1994; Milani, 1941; Niimi et al., 1999) while loss of ommatidial clusters and a mild roughening of the eye are seen with weak optix hypomorphic alleles (null mutants have not been described; Weasner and Kumar, unpublished data). Forced expression of either gene is capable of redirecting the fates of nonretinal tissues toward that of an eye (Seimiya and Gehring, 2000; Weasner et al., 2007). However, it is unlikely that the exact same biochemical mechanisms underlie So and Optix activities because differences in binding partner selection have been described for these proteins (Kenyon et al., 2005a,b).

Like all SIX proteins, So and Optix are characterized by the presence of two evolutionarily conserved functional motifs: a homeobox for nucleic acid recognition and a SIX domain for protein-protein interactions. Two recent studies have shown that both proteins bind to DNA sequences containing a common recognition motif (Berger et al., 2008; Noyes et al., 2008). In contrast, each protein has been shown to interact with very distinct sets of protein partners through the SIX domains (Kenyon et al., 2005a,b). Consistent with these molecular features, a functional dissection of the two proteins using domain deletions and chimeras demonstrated that only the homeodomains are functionally interchangeable (Weasner et al., 2007).

The same study also demonstrated that the C-terminal (CT) segments of So and Optix, which share virtually no homology with each other, may, in addition, serve to functionally distinguish one SIX protein from another. First, in rescue assays the CT regions were shown to be incapable of substituting for each other. Second, the CT of Optix appears to harbor inhibitory or repressive activity. When either wild type Optix or a So/Optix chimera containing the Optix CT is expressed in the eye, retinal development is blocked. A comparison of the CT segments of Optix and its two murine homologs, Six 3 and Six6, identified two zones of conservation (Weasner et al., 2007). One of these two regions lies directly adjacent to the homeodomain and has been shown to serve as an extension of this DNA binding domain. The role of this segment appears not to be in DNA binding specificity but rather in increasing the binding affinity of the homeodomain to DNA (Hu et al., 2008).

In this report, we further investigate the Optix CT segment and identify regions that together are required for the observed inhibitory activity. We show that this activity also depends upon 
the ability of Optix to bind DNA and interact with its normal complement of protein binding partners. We also demonstrate that the CT is required for other functions of Optix including the generation of ectopic eyes in nonretinal tissues. It thus appears that the functional specificity of SIX proteins is determined by direct protein-protein interactions via the SIX domain and through the use of the class-specific CT segments. Finally, we show that several vertebrate SIX proteins are capable of functioning during normal Drosophila eye development despite having not been expressed within the eye of any organism for several hundred million years. Interestingly, these same genes appear to have lost the ability to induce ectopic eye formation suggesting that there are inherent differences between initiating retinal development within the normal eye primordium and transforming nonretinal epithelia into eye tissue.

\section{MATERIALS AND METHODS}

\section{Fly Stocks, Microscopy, and DNA constructs}

The following fly stocks were used in this manuscript: ey-GAL4 (gift of Walter Gehring), GMR-GAL4 (gift of Larry Zipursky), dpp-GAL4 (gift of Janice Fischer), UAS-optix (Weasner et al., 2007), cb49-GAL4, rn-GAL4, c309-GAL4, c253-GAL4 (gifts of the Bloomington Stock Center). All GAL4 crosses were conducted at $25^{\circ} \mathrm{C}$. Adult eyes were prepared for SEM by dehydration through an ethanol gradient followed by an incubation in 50:50 ethanol:HMDS and finally by passage through $100 \%$ HMDS. An Optix cDNA is the gift of Walter Gehring and the Six 1-6 cDNAs are gifts of Richard Maas.

\section{Generation of UAS Lines}

We used PCR and the Gateway Cloning System from Invitrogen to clone mammalian Six 1-6 genes into the pUAST expression vector. Similar methods were used to generate molecules that encode Optix protein variants. Primer sequences and cloning steps are available upon request. Stable germline transformants were generated using standard methods (Rubin and Spradling, 1983). Multiple independent transformants were generated and tested for each construct.

The limits of the Optix proteins were obtained from published reports (Seimiya and Gehring, 2000). The Optix protein is 487 amino acids in length and can be divided into four regions: the N-terminal (NT) includes amino acids 1-36, the SIX domain (SD) includes amino acids 37-155, the homeobox DNA binding domain (HD) includes amino acids 156-215, and the Cterminal (CT) includes amino acids 216-487. The CT region was further subdivided into five regions: region A includes amino acids 216-222, region B includes amino acids 223-343, region $\mathrm{C}$ includes amino acids 344-354, region $\mathrm{D}$ includes amino acids $355-419$, region $\mathrm{E}$ includes amino acids 420-487. It should be noted that regions $\mathrm{D}$ and $\mathrm{E}$ are conserved just within the Drosophilids while regions A and C are conserved in other insects and in vertebrate species such as the mouse and humans. The subdomains of Optix are diagrammed in Figures 1 and 2.

The following deletion constructs were constructed and used in this report: Optix $\triangle \mathrm{NT}$ consists of amino acids 37-487, Optix $\Delta$ SD consists of amino acids 1-36 fused to resides 156-487, Optix $\Delta \mathrm{HD}$ consists of amino acids 1-155 fused to residues 216-487, Optix $\Delta \mathrm{CT}$ consists of amino acids 1-215, Optix $\Delta$ CT-A consists of amino acids 1-215 fused to residues 223-487, Optix $\Delta$ CT-B consists of amino acids 1-222 fused to residues 344-419, Optix $\Delta$ CT-C consists of amino acids 1-343 fused to residues 355-487, Optix $\triangle \mathrm{CT}$-D+E consists of amino acids 1-351, Optix $\Delta$ CT-E consists of amino acids 1-419. These constructs are diagrammed in Figures 1 and 2 .

Individual subsections of the CT (except for region B) were fused to the Optix backbone comprised of amino acids 1-215: Optix CT-A contains amino acids 1-283 (note that this 
construct contains a portion of region B), Optix CT-C contains amino acids 1-215 fused to residues 344-354, Optix CT-D contains amino acids 1-215 fused to residues 355-419, and Optix CT-A+C+D contains amino acids 1-222 fused to residues 344-419. We also fused the entire CT segment (amino acids 216-487) to the entire open reading frame of GFP to create GFP-Optix CT (note that this construct has includes a nuclear localization sequence [NLS]).

\section{Orthologous Sequence Search}

Individual subregions of the Optix CT segment from Drosophila melanogaster was used as reference sequence for input into searches against other sequenced and annotated

Drosophila genomes as well as the genomes of the honeybee, mosquito, flour beetle, silkworm, mouse, and human. The nucleotide sequences were obtained from exhaustive searches on NCBI and on Flybase (Altschul et al., 1997). Putative homologous sequences to each region of the Optix CT of D. melanogaster Optix were identified in D. simulans, D. sechellia, D. yakuba, D. erecta, D. ananassae, D. pseudoobscura, D. persimilis, D. wilisoni, D. mojavensis, D. virilis, and D. grimshawi.

\section{RESULTS}

\section{The C-Terminal Segment of Optix Harbors Inhibitory Activity}

Optix, like all SIX family members, is defined by the presence of an evolutionarily conserved protein-protein interaction domain (SIX domain is abbreviated SD) and a homeobox DNA binding motif (HD). These functional domains are flanked by two nonconserved regions that are generically referred to as the N-terminal (NT) and C-terminal (CT) segments (Fig. 1a). Forced expression of optix within the developing eye via the glass eye specific enhancer, which directs expression in all cells posterior to the furrow (GMR, Hay et al., 1994), is sufficient to drastically inhibit eye development (Fig. 1b; Weasner et al., 2007). Expression of the other two SIX genes, so and DSix4, do not have as severe inhibitory effects on the eye when overexpressed (data not shown). When the eyeless eye specific enhancer, which activates transcription ahead of the morphogenetic furrow (Hauck et al., 1999), directs optix expression we observe dramatic reductions in eye formation (data not shown). In an effort to gain a better understanding of Optix function we set out to determine if this inhibitory activity could be mapped to a specific portion of the Optix protein.

To this end we generated a series of Optix protein deletions, expressed them in the developing retina via GMR-GAL4, and then assayed the effect of each deletion molecule on adult eye structure (Fig. 1a,c-f). For each construct depicted in Figure 1a multiple insertion lines were tested. It appears that the only region of the protein not required for inhibition is the nonconserved N-terminal segment (Fig. 1c). Of the remaining regions, release of inhibition follows the removal of either the SD (Optix $\Delta \mathrm{SD}$, Fig. 1d) or the HD (Optix $\Delta$ HD, Fig. 1e). This result is easily explained because several known protein-protein interactions would be abolished with the former construct while the latter construct would be unable to bind to DNA. The requirement for the CT segment (Fig. 1f) is more intriguing as no function has been ascribed to this portion of the protein and virtually no sequence conservation exists among the different subclasses of SIX proteins across this segment. The necessity of the CT is supported by earlier observations that replacement of the CT region of Sine Oculis with that of Optix (So/ Optix CT) is sufficient to severely inhibit eye development to the level of full length Optix while additional So/Optix chimeras in which the other protein domains were exchanged did not have the same effect (Weasner et al., 2007). We expressed the Optix CT alone (fused to a nuclear localization sequence) and saw no visible alterations in the developing eye (data not shown). It appears that the CT is corequired with both SD protein-protein interaction and HD DNA binding motifs (Fig. 1d,e). We conclude from these experiments that the Optix CT region itself is necessary but not sufficient for the observed inhibitory effect. 


\section{Functional Dissection of the Optix C-Terminal Segment}

A comparison between Optix and mammalian Six 3 and Six6 proteins, two SIX family members that fall within the Optix subclass, indicates that there is limited homology within the CT region at two positions (Fig. 2a, Weasner et al., 2007). One of these segments (designated as region A) has been shown to increase the affinity of the HD to DNA thereby acting as a C-terminal extension of the HD (Hu et al., 2008). In contrast, no functional role has been assigned to the second region of conservation (Fig. 2a; designated as region C). These two segments are separated by a stretch of nonconserved amino acids (designated as region B). Finally, we arbitrarily separated the distal portion of the protein into two segments (designated as regions $\mathrm{D}$ and $\mathrm{E}$ ). To determine which region(s) of the Optix CT contributes to the inhibitory activity, we systematically removed each one and expressed each resulting deletion construct in the developing retina and assayed the effect on adult eye structure (Fig. 2b-g). For each construct depicted in Figure $2 \mathrm{~b}$ multiple insertion lines were tested. Regions A and D appear to be absolutely required as expression of constructs lacking these segments (Optix $\Delta$ CT-A and Optix $\Delta$ CT-D+E) does not inhibit eye development. In both cases, the eye is nearly wild type and resembles constructs lacking the entire $\mathrm{CT}$ region (Figs. 1f and 2b,c,f). However, region $\mathrm{C}$ is only partially required as its removal (Optix $\Delta \mathrm{CT}-\mathrm{C}$ ) partially alleviates, but does not eliminate, the roughening of the adult eye (Fig. 2b,e). Finally, regions B and E appear to be completely dispensable as the expression of constructs lacking these regions, Optix $\Delta \mathrm{CT}$-B and Optix $\Delta \mathrm{CT}$-E, still induces a rough eye almost identical to that caused by expression of full-length Optix (Figs. 1B and 2b,d,g). We conclude from these results that the inhibitory activity of the $\mathrm{CT}$ resides within the conserved regions $\mathrm{A}$ and $\mathrm{C}$ and in the nonconserved region D.

As we have been able to localize the inhibitory activity of the CT to distinct subregions, we set out to determine if the inhibitory activity of the CT can be recapitulated by the expression of these individual regions or whether it is dependent upon the combinatorial use of these segments. Expression of the Optix core protein fused to individual segments failed to induce a rough eye (data not shown). Instead, a construct in which the core protein and all three regions (A, C, D) were present is sufficient to block eye development (Fig. 2b,d).

Interestingly, region D is not conserved between Optix, Six3, and Six6. We sought to determine if this difference influences the ability of either vertebrate homolog to induce a rough eye to the same degree as Optix. Expression of Six 3 and Six6 inhibits eye development but not nearly as severely as that of Optix (Figs. 1B and 3a,b). One potential explanation, among several, is that region $\mathrm{D}$ has inhibitory activity that further distinguishes Optix from the vertebrate members of the subgroup. Supporting this contention is the observation that region D is conserved in Optix orthologs within nearly all sequenced Drosophila species (see Fig. 4). Interestingly, this region appears to be specific to the Drosophilids as we do not find it in any other insect including the flour beetle, mosquito, honeybee, or silkworm.

We then looked at how well the other regions within the CT are conserved. We calculated the overall sequence conservation by making pairwise alignments between $D$. melanogaster and each Drosophila species, the flour beetle, mosquito, honeybee and silkworm. The pairwise conservation values were then averaged to produce overall sequence conservation values. Among the Drosophilids, region $\mathrm{A}=100 \%$, region $\mathrm{B}=86 \%$, region $\mathrm{C}=95 \%$, region $\mathrm{D}=90 \%$, and region $\mathrm{E}=91 \%$. The high level of conservation with the Drosophilids is likely due to the short evolutionary distance that separates each species. By extending our analysis to the other basal insects and thus a greater evolutionary distance we discovered that only two regions were conserved (region $\mathrm{A}=63 \%$ and region $\mathrm{C}=36 \%$ ). The other sections, including region $\mathrm{D}$, have virtually no homology outside the Drosophilds. As region D appears restricted to the Drosophilids but has no homology in the other insects or higher organisms suggests that its activity is specific to the Drosophilids. 


\section{The C-terminal Segment of Optix is Required for Ectopic Eye Formation}

Because of the unexpected requirement for the Optix CT in inhibiting eye development we set out to determine if this portion of the protein was also required for other functions including the formation of ectopic eyes. Expression of full-length Optix protein in developing imaginal discs via the UAS/GAL4 system is sufficient to induce ectopic eyes in the developing antenna and wings (Seimiya and Gehring, 2000; Weasner et al., 2007). The CT domain is required for ectopic eye formation as retinal development is not induced within these tissues in response to expression of Optix $\Delta \mathrm{CT}$ (Fig. 5a). We set out to see if the CT of Six 3 and Six6 are also required for the activities of these proteins. However, none of the vertebrate homologs (Sixl-6) are capable of inducing ectopic eye formation in flies therefore we were unable to make the appropriate comparison (Fig. 5a). Interestingly, Six6 was capable of producing an extra-eye field adjacent to the ventral quadrant of the normal eye when expressed with dpp-GAL4, which is expressed along the posterior-lateral edges of the eye field and within a pie shaped zone of the ventral antenna (Fig. 5b,c). These fields differ from ectopic eyes in that they arise from regions immediately surrounding the eye field that already express most, if not all, members of the retinal determination network but are normally fated to become head cuticle. In some circumstances these extra eye fields appear as separate entities (Fig. 5b) while in others it is clear that they are part of the endogenous eye field (Fig. 5c). Extra eye fields, similar in location, are also generated by the forced expression of Pax6(5a) homologs eyegone (eyg) and twin of eyegone (toe; Jang et al., 2003; Yao et al., 2008).

\section{Functional Conservation During Eye Development is Restricted to the So and DSix4 Subgroups}

Within Drosophila, so plays a much greater role than optix within the visual system. It is expressed and governs the specification of the compound eye and ocelli (Cheyette et al., 1994; Serikaku and O'Tousa, 1994). It plays additional roles in the specification and maintenance of photoreceptor cell fates (Pignoni et al., 1997). Weak hypomorphic loss-offunction optix mutants have very mild phenotypic effects including the loss of ommatidia (Weasner and Kumar, unpublished observation), which suggests that it functions during early eye development. However, the lack of optix null mutants has made it difficult to completely ascertain the role that it may play in the fly retina. Interestingly, within the vertebrate retina, Six3 and Six6 are the main players while the homologs of so, Sixl and Six2, are not expressed (Bovolenta et al., 1998; Gallardo et al., 1999; Ghanbari et al., 2001; Granadino et al., 1999; Jean et al., 1999; Leppert et al., 1999; Oliver et al., 1995; Toy and Sundin, 1999; Zhou et al., 2000). This switch in SIX protein usage within the retina prompted us to investigate which vertebrate SIX genes could substitute for so during normal eye development. To this end we individually expressed Six 1-6 in retinas harboring an eye specific so loss-of-function mutation $\left(\mathrm{so}^{1}\right.$; Fig. 5e) and assayed the ability of each construct to restore retinal development. For each vertebrate construct multiple insertion lines were tested. Although vertebrate Six1 and Six2 have not been expressed within the retina for several hundred million years, activation of either gene in the fly retina is sufficient to restore the eye nearly back to wild type indicating that both genes have most likely retained the ability (through protein-protein interactions and regulating transcriptional targets) to function within the context of eye development (Fig. 5d,f). Six3 and Six6, like optix itself, failed to support eye development (Fig. 5d, data not shown). This provides further evidence that members of the So and Optix subgroups regulate development through distinct mechanisms. Quite surprising is the fact that Six4 was able to partially restore eye development to $s o^{l}$ mutants (Fig. $5 \mathrm{~d}, \mathrm{~g}$ ). Even more remarkable is the fact that DSix4, the fly homolog of vertebrate Six4, is capable of rescuing so ${ }^{I}$ mutants to the same levels as members of the So subgroup (Fig. 5d, data not shown). This was unexpected because DSix4 is not expressed in the retina, but rather is expressed and functions in the developing gonad and other mesodermal derivatives (Clark et al., 2006; Kirby et al., 2001). It suggests that DSix4 and So interact with similar if not identical protein partners and regulate a common 
set of transcriptional targets within the context of eye development. It is not yet clear if So can substitute for DSix4 in the developing gonad.

\section{DISCUSSION}

The SIX family of homeobox transcription factors plays important roles in many developmental contexts including retinal determination (reviewed in Kumar, 2008). In parsing the responsibilities of these proteins in development, attention has been largely placed on two evolutionarily conserved domains: the SIX protein-protein interaction domain and the homeobox DNA binding domain. However, it appears that the nonconserved segments that flank the SIX-HD core also contain information that is relevant for the function of these transcription factors. For instance, the nonconserved N-terminal region of human Six 1 mediates interactions with the APC ${ }^{\mathrm{cdh} 1}$ protein destruction complex (Christensen et al., 2007). It is also known that Casein Kinase 2 phosphorylates hSix 1 at sites located within the C-terminal tail (Ford et al., 2000). These two modifications play important roles in the stability and activity of hSix 1 . Additionally, the C-terminal tails of Six 2 and Six 4 contain transcriptional activation domains indicating that, under certain circumstances, these proteins can directly activate transcription in the absence of interactions with transcriptional coactivators (Brodbeck and Englert, 2004; Kawakami et al., 1996a,b). Finally, a set of amino acids within the Cterminus of Six6 lies adjacent to the homeodomain has been shown to be necessary and sufficient to increase the affinity of the homeodomain to DNA (Hu et al., 2008).

We have previously shown that forced expression of Optix in the developing eye leads to severe alterations in eye structure (Weasner et al., 2007). This activity was mapped to the nonconserved C-terminal tail as the replacement of the So CT with that of Optix leads to the same degree of roughening as full-length Optix. In this report, we have extended this early observation by functionally dissecting the C-terminal segment of the Optix protein and (1) demonstrated that while the $\mathrm{CT}$ is necessary for inhibiting eye development, it also requires the activity of the protein-protein interaction and DNA binding regions; (2) identified that regions $\mathrm{A}, \mathrm{C}$, and $\mathrm{D}$ of the $\mathrm{CT}$ are required for blocking retinal formation; (3) shown that region $\mathrm{D}$ is specifically conserved just within the Drosophilids; (4) demonstrated that the CT is required for the ability of Optix to induce ectopic eyes; (5) shown that none of the vertebrate SIX proteins are capable of inducing ectopic eyes in flies; and (6) demonstrated that in addition to so homologs, expression of DSix4 and Six4 can also rescue $s o^{1}$ loss-of-function mutants.

As optix null mutants are unavailable we cannot definitively demonstrate that the CT is required for Optix function during normal eye development. However, data from a functional dissection of the So protein may shed light on this question. In these forced expression experiments, removal of the CT renders the truncated So protein incapable of inducing ectopic eyes.

However, the loss of the CT does not interfere with the ability of the mutant protein to rescue eye development in loss-of-function mutants (Weasner et al., 2007). If this were to hold true for Optix then it would suggest that a common mechanism might underlie the activities of these two proteins. One possibility is that the CT functions redundantly by contributing putative activation and/or repression domains. These activities are normally specified by the interactions with transcriptional coactivators and corepressors through the SIX domain. For example, So binds to the transcriptional coactivator Eya (Pignoni et al., 1997) and Optix binds to the corepressor Groucho (Gro; Kenyon et al., 2005a,b). Similar interactions are reported for the vertebrate homologs (reviewed by Kumar, 2008). During normal eye development, the SIX proteins may function primarily through these protein-protein interactions thus deletions of the CT may have little to no effect. In contrast, in situations where the SIX proteins are forcibly expressed outside of their normal domains (i.e., behind the furrow for optix and throughout other imaginal discs for both so and optix), the traditional binding partners may not be expressed. In these situations, potential intrinsic activation and repression domains (like those 
seen with Six 2 and Six4) may exist within the CT and could substitute, at least partially. Thus their removal prevents the formation of ectopic eyes or the inhibition of eye development.

The demonstration that Drosophila DSix 4 and murine Six1, Six2, and Six 4 can rescue the noeye phenotype of Drosophila so ${ }^{l}$ mutants allows us to predict that these four proteins most likely regulate the same transcriptional targets and are able to interact with the same set of proteins as So. This is remarkable as DSix4, Sixl, and Six2 are not expressed within the developing eye and have not been for several hundred million years (Clark et al., 2006; Kirby et al., 2001; Oliver et al., 1995) and while Six4 is expressed in the retina, to date no eye abnormalities have been associated with loss-of-function mutations (Ghanbari et al., 2001; Kawakami et al., 1996a,b; Niya et al., 1998). Why then are none of these SIX proteins capable of inducing ectopic eye formation in flies? It may be that the intrinsic activation and repression activities of these proteins are not identical to those of the fly proteins. One potential source of such differences may in fact be the nonconserved C-terminal regions.

\section{ACKNOWLEDGMENTS}

We would like to thank Bonnie M. Weasner and Claire L. Salzer for comments on the manuscript and Richard Maas, Walter Gehring, Janice Fischer, Larry Zipursky and the Bloomington Drosophila Stock Center for gifts of cDNA clones and fly strains.

Contract grant sponsor: National Institutes of Health, Contract grant number: R01 EY014863

\section{LITERATURE CITED}

Altschul SF, Madden TL, Schaffer AA, Zhang J, Zhang Z, Miller W, Lipman DJ. Gapped BLAST and PSI-BLAST: A new generation of protein database search programs. Nucleic Acids Res 1997;25:3389-3402. [PubMed: 9254694]

Bessa J, Gebelein B, Pichaud F, Casares F, Mann RS. Combinatorial control of Drosophila eye development by eyeless, homothorax, and teashirt. Genes Dev 2002;16:2415-2427. [PubMed: 12231630]

Bonini NM, Leiserson WM, Benzer S. The eyes absent gene: Genetic control of cell survival and differentiation in the developing Drosophila eye. Cell 1993;72:379-395. [PubMed: 8431945]

Bovolenta P, Mallamaci A, Puelles L, Boncinelli E. Expression pattern of cSix3, a member of the Six/ sine oculis family of transcription factors. Mech Dev 1998;70:201-203. [PubMed: 9510037]

Brodbeck S, Englert C. Genetic determination of nephrogenesis: The Pax/Eya/Six gene network. Pediatr Nephrol 2004;19:249-255. [PubMed: 14673635]

Cheyette BN, Green PJ, Martin K, Garren H, Hartenstein V, Zipursky SL. The Drosophila sine oculis locus encodes a homeodomain-containing protein required for the development of the entire visual system. Neuron 1994;12:977-996. [PubMed: 7910468]

Christensen KL, Brennan JD, Aldridge CS, Ford HL. Cell cycle regulation of the human Six 1 homeoprotein is mediated by APC(Cdh1). Oncogene 2007;26:3406-3414. [PubMed: 17130831]

Clark IB, Boyd J, Hamilton G, Finnegan DJ, Jarman AP. D-six4 plays a key role in patterning cell identities deriving from the Drosophila mesoderm. Dev Biol 2006;294:220-231. [PubMed: 16595131]

Cohen, SM. Imaginal disc development. In: Bate, M.; Arias, AM., editors. Cold Spring Harbor Laboratory Press; Cold Spring Harbor, NY: 1993. p. 747-841.

Czerny T, Halder G, Kloter U, Souabni A, Gehring WJ, Busslinger M. Twin of eyeless, a second Pax-6 gene of Drosophila, acts upstream of eyeless in the control of eye development. Mol Cell 1999;3:297307. [PubMed: 10198632]

Ford HL, Landesman-Bollag E, Dacwag CS, Stukenberg PT, Pardee ABSeldin DC. Cell cycle-regulated phosphorylation of the human SIX1 homeodomain protein. J Biol Chem 2000;275:22245-22254. [PubMed: 10801845]

Gallardo ME, Lopez-Rios J, Fernaud-Espinosa I, Granadino B, Sanz R, Ramos C, Ayuso C, Seller MJ, Brunner HG, Bovolenta P, et al. Genomic cloning and characterization of the human homeobox gene 
SIX6 reveals a cluster of SIX genes in chromosome 14 and associates SIX6 hemizygosity with bilateral anophthalmia and pituitary anomalies. Genomics 1999;61:82-91. [PubMed: 10512683]

Ghanbari H, Seo HC, Fjose A, Brandli AW. Molecular cloning and embryonic expression of Xenopus Six homeobox genes. Mech Dev 2001;101:271-277. [PubMed: 11231090]

Granadino B, Gallardo ME, Lopez-Rios J, Sanz R, Ramos C, Ayuso C, Bovolenta P, de Cordoba SR. Genomic cloning, structure, expression pattern, and chromosomal location of the human SIX3 gene. Genomics 1999;55:100-105. [PubMed: 9889003]

Hauck B, Gehring WJ, Walldorf U. Functional analysis of an eye specific enhancer of the eyeless gene in Drosophila. Proc Natl Acad Sci USA 1999;96:564-569. [PubMed: 9892673]

Hay BA, Wolff T, Rubin GM. Expression of baculovirus P35 prevents cell death in Drosophila. Development 1994;120:2121-2129. [PubMed: 7925015]

Held, LI. Developmental and Cell Biology Series 39. Cambridge University Press; Cambridge: 2002. Imaginal discs: The genetic and cellular logic of pattern formation; p. 460

Hu S, Mamedova A, Hegde RS. DNA-binding and regulation mechanisms of the SIX family of retinal determination proteins. Biochemistry 2008;47:3586-3594. [PubMed: 18293925]

Jang CC, Chao JL, Jones N, Yao LC, Bessarab DA, Kuo YM, Jun S, Desplan C, Beckendorf SK, Sun YH. Two Pax genes, eye gone and eyeless, act cooperatively in promoting Drosophila eye development. Development 2003;130:2939-2951. [PubMed: 12756177]

Jean D, Bernier G, Gruss P. Six6 (Optx2) is a novel murine Six3-related homeobox gene that demarcates the presumptive pituitary/hypothalamic axis and the ventral optic stalk. Mech Dev 1999;84:31-40. [PubMed: 10473118]

Kawakami K, Ohto H, Ikeda K, Roeder RG. Structure, function and expression of a murine homeobox protein AREC3, a homologue of Drosophila sine oculis gene product, and implication in development. Nucleic Acids Res 1996a;24:303-310. [PubMed: 8628654]

Kawakami K, Ohto H, Takizawa T, Saito T. Identification and expression of six family genes in mouse retina. FEBS Lett 1996b;393:259-263. [PubMed: 8814301]

Kenyon KL, Li DJ, Clouser C, Tran S, Pignoni F. Fly SIX-type homeodomain proteins Sine oculis and Optix partner with different cofactors during eye development. Dev Dyn 2005a;234:497-504. [PubMed: 15937930]

Kenyon KL, Yang-Zhou D, Cai CQ, Tran S, Clouser C, Decene G, Ranade S, Pignoni F. Partner specificity is essential for proper function of the SIX-type homeodomain proteins Sine oculis and Optix during fly eye development. Dev Biol 2005b;286:158-168. [PubMed: 16125693]

Kirby RJ, Hamilton GM, Finnegan DJ, Johnson KJ, Jarman AP. Drosophila homolog of the myotonic dystrophy-associated gene, SIX5, is required for muscle and gonad development. Curr Biol 2001;11:1044-1049. [PubMed: 11470409]

Kumar JP. The sine oculis homeobox (SIX) family of transcription factors as regulators of development and disease. Cell Mol Life Sci. 2008in press

Kumar JP, Moses K. EGF receptor and Notch signaling act upstream of Eyeless/Pax6 to control eye specification. Cell 2001;104:687-697. [PubMed: 11257223]

Leppert GS, Yang JM, Sundin OH. Sequence and location of SIX3, a homeobox gene expressed in the human eye. Ophthalmic Genet 1999;20:7-21. [PubMed: 10415461]

Mardon G, Solomon NM, Rubin GM. Dachshund encodes a nuclear protein required for normal eye and leg development in Drosophila. Development 1994;120:3473-3486. [PubMed: 7821215]

Milani R. Two new eye-shape mutant alleles in Drosophila melanogaster. D. I. S 1941;14:52.

Niimi T, Seimiya M, Kloter U, Flister S, Gehring WJ. Direct regulatory interaction of the eyeless protein with an eye-specific enhancer in the sine oculis gene during eye induction in Drosophila. Development 1999;126:2253-2260. [PubMed: 10207149]

Niiya A, Ohto H, Kawakami K, Araki M. Localization of Six4/AREC3 in the developing mouse retina; implications in mammalian retinal development. Exp Eye Res 1998;67:699-707. [PubMed: 9990334]

Noyes MB, Christensen RG, Wakabayashi A, Stormo GD, Brodsky MH, Wolfe SA. Analysis of homeodomain specificities allows the family-wide prediction of preferred recognition sites. Cell 2008;133:1277-1289. [PubMed: 18585360] 
Oliver G, Wehr R, Jenkins NA, Copeland NG, Cheyette BN, Hartenstein V, Zipursky SL, Gruss P. Homeobox genes and connective tissue patterning. Development 1995;121:693-705. [PubMed: 7720577]

Pai CY, Kuo TS, Jaw TJ, Kurant E, Chen CT, Bessarab DA, Salzberg A, Sun YH. The homothorax homeoprotein activates the nuclear localization of another homeoprotein, extradenticle, and suppresses eye development in Drosophila. Genes Dev 1998;12:435-446. [PubMed: 9450936]

Pan D, Rubin GM. Targeted expression of teashirt induces ec-topic eyes in Drosophila. Proc Natl Acad Sci USA 1998;95:15508-15512. [PubMed: 9860999]

Pignoni F, Hu B, Kenton HZ, Xiao J, Garrity PA, Zipursky SL. The eye-specification proteins So and Eya form a complex and regulate multiple steps in Drosophila eye development. Cell 1997;91:881891. [PubMed: 9428512]

Quiring R, Walldorf U, Kloter U, Gehring WJ. Homology of the eyeless gene of Drosophila to the small eye gene in mice and Aniridia in humans [see comments]. Science 1994;265:785-789. [PubMed: 7914031]

Ready DF, Hanson TE, Benzer S. Development of the Drosophila retina, a neurocrystalline lattice. Dev Biol 1976;53:217-240. [PubMed: 825400]

Rubin GM, Spradling AC. Vectors for P element mediated gene transfer in Drosophila. Nucl Acids Res 1983;11:6341-6351. [PubMed: 6312420]

Seimiya M, Gehring WJ. The Drosophila homeobox gene optix is capable of inducing ectopic eyes by an eyeless-independent mechanism. Development 2000;127:1879-1886. [PubMed: 10751176]

Serikaku MA, O'Tousa JE. Sine oculis is a homeobox gene required for Drosophila visual system development. Genetics 1994;138:1137-1150. [PubMed: 7896096]

Singh A, Kango-Singh M, Sun YH. Eye suppression, a novel function of teashirt, requires wingless signaling. Development 2002;129:4271-4280. [PubMed: 12183379]

Toy J, Sundin $\mathrm{OH}$. Expression of the optx 2 homeobox gene during mouse development. Mech Dev 1999;83:183-186. [PubMed: 10381579]

Weasner B, Salzer C, Kumar JP. Sine oculis, a member of the SIX family of transcription factors, directs eye formation. Dev Biol 2007;303:756-771. [PubMed: 17137572]

Wolff T, Ready DF. The beginning of pattern formation in the Drosophila compound eye: The morphogenetic furrow and the second mitotic wave. Development 1991;113:841-850. [PubMed: 1726564]

Yao JG, Weasner BM, Wang LS, Jang CC, Weasner B, Tang CY, Salzer CL, Chen CH, Hay BA, Sun $\mathrm{YH}$, et al. Differential requirements for the Pax6(5a) genes eyegone and twin of eye-gone during eye development in Drosophila. Dev Biol 2008;315:535-551. [PubMed: 18275947]

Zhou X, Hollemann T, Pieler T, Gruss P. Cloning and expression of xSix3, the Xenopus homologue of murine Six3. Mech Dev 2000;91:327-330. [PubMed: 10704858] 
a

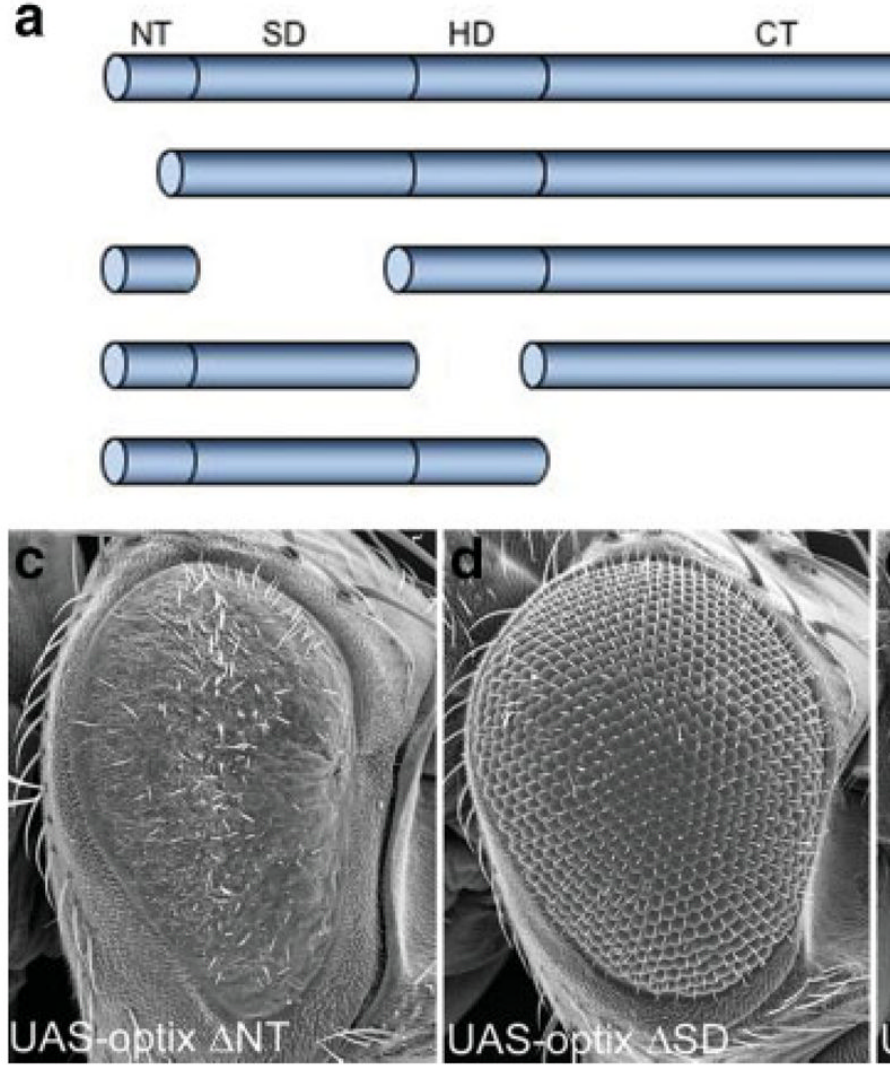

HD

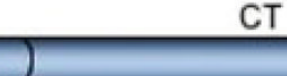

CT

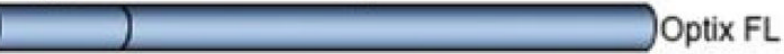

Optix $\Delta N T$

Optix $\Delta S D$

Optix $\triangle H D$

Optix $\Delta C T$

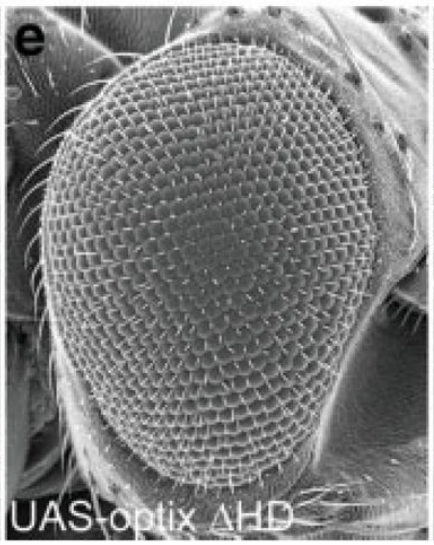

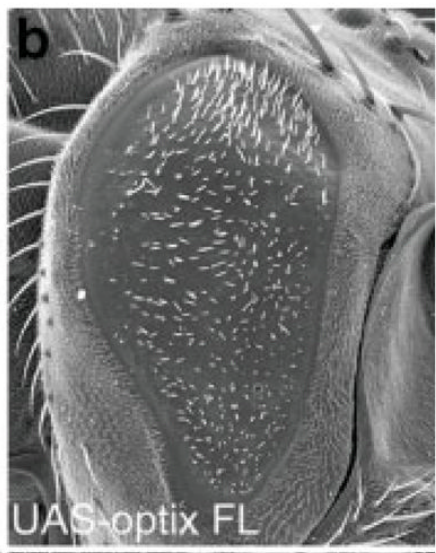

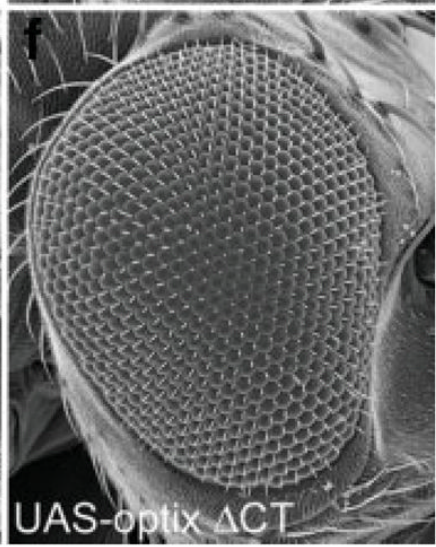

FIG. 1.

Inhibition of eye development by forced expression of Optix requires the nonconserved CT segment. (a) Schematic drawing of Optix full-length and Optix deletion constructs. (b-f) Scanning electron micrographs of adult compound eyes. The identity of individual UAS constructs is listed in the bottom left of each panel. All constructs were expressed in developing photoreceptor cells via the GMR-GAL4 driver. Anterior is to the right. 


\section{a}
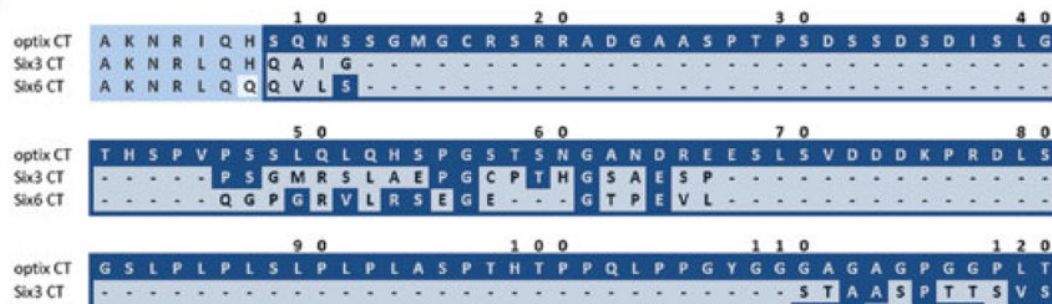

$\sec \mathrm{CT}$
$\operatorname{sac} 6 \mathrm{CT}$

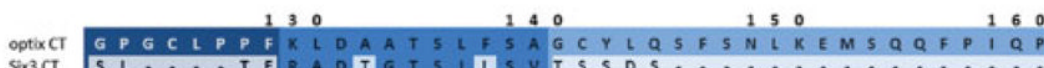

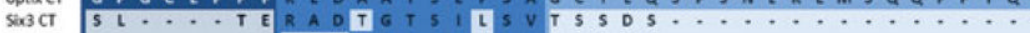

$\sec 60$

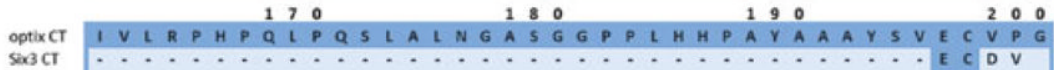

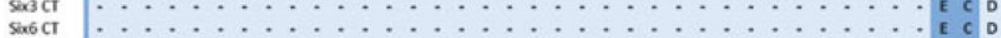

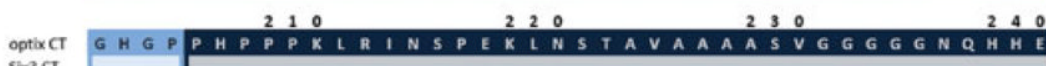

$\operatorname{sen} 3 \mathrm{CT}$
$\operatorname{sen} 6 \mathrm{CT}$

optix C

$\mathrm{ser} 3 \mathrm{CT}$

$\operatorname{sen} 6 \mathrm{CI}$

\section{b}
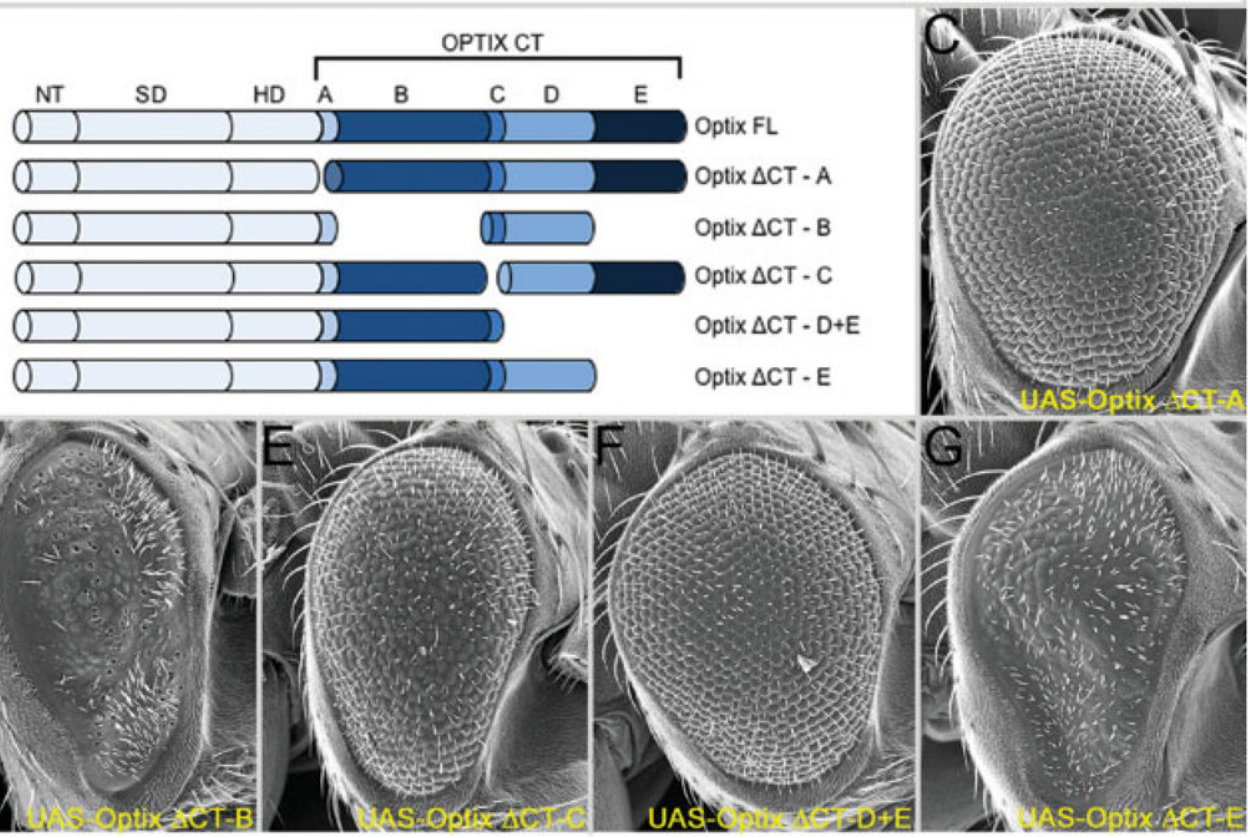

FIG. 2.

Functional dissection of the Optix CT reveals a requirement for several subdomains. (a) Sequence alignment of the Optix, Six3, and Six6 CT regions. (b) Schematic diagram of CT deletion constructs. (c-g) Scanning electron micrographs of adult compound eyes. The identity of individual UAS constructs is listed below each panel. All constructs were expressed in developing photoreceptor cells via the GMRGAL4 driver. A color key for panels A and B can be found in the right side of panel B. Anterior is to the right. 

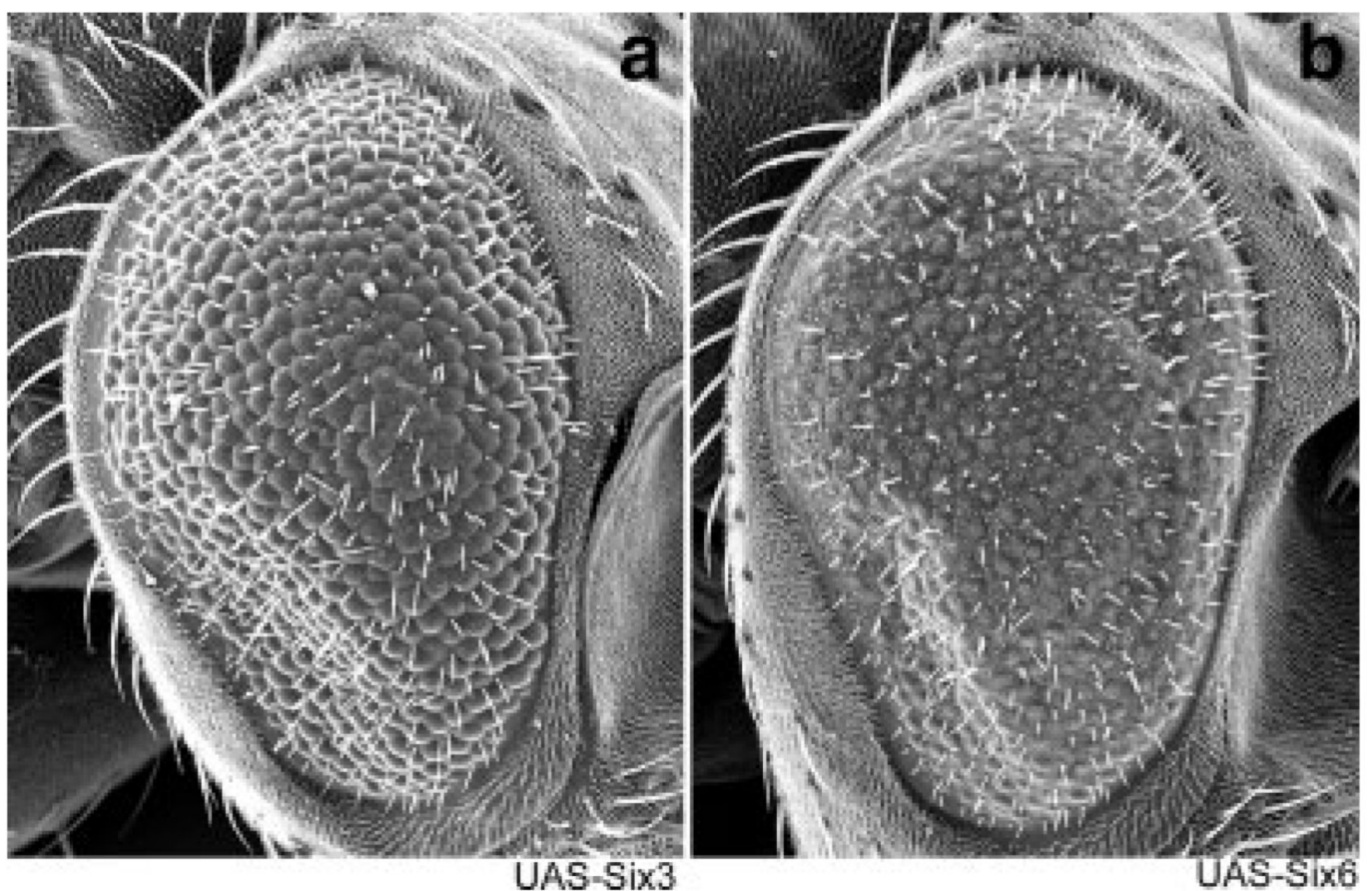

FIG. 3.

Inhibition of eye development by Six 3 and Six6. (a,b) Scanning electron micrographs of adult compound eyes. The identity of individual UAS constructs is listed below each panel. All constructs were expressed in developing photoreceptor cells via the GMRGAL4 driver. Anterior is to the right. 
D. melanogaster

D. simulans

D. sechellia

D. yakuba

D. erecta

D. ananassae

D. pseudoobscura

D. persimilis

D. wilistoni

D. mojavensis

D. virilis

D. grimshawi

D. melanogaster

D. simulans

D. sechellia

D. yakuba

D. erecta

D. ananassae

D. pseudoobscura

D. persimilis

D. wilistoni

D. mojavensis

D. virilis

D. grimshawi

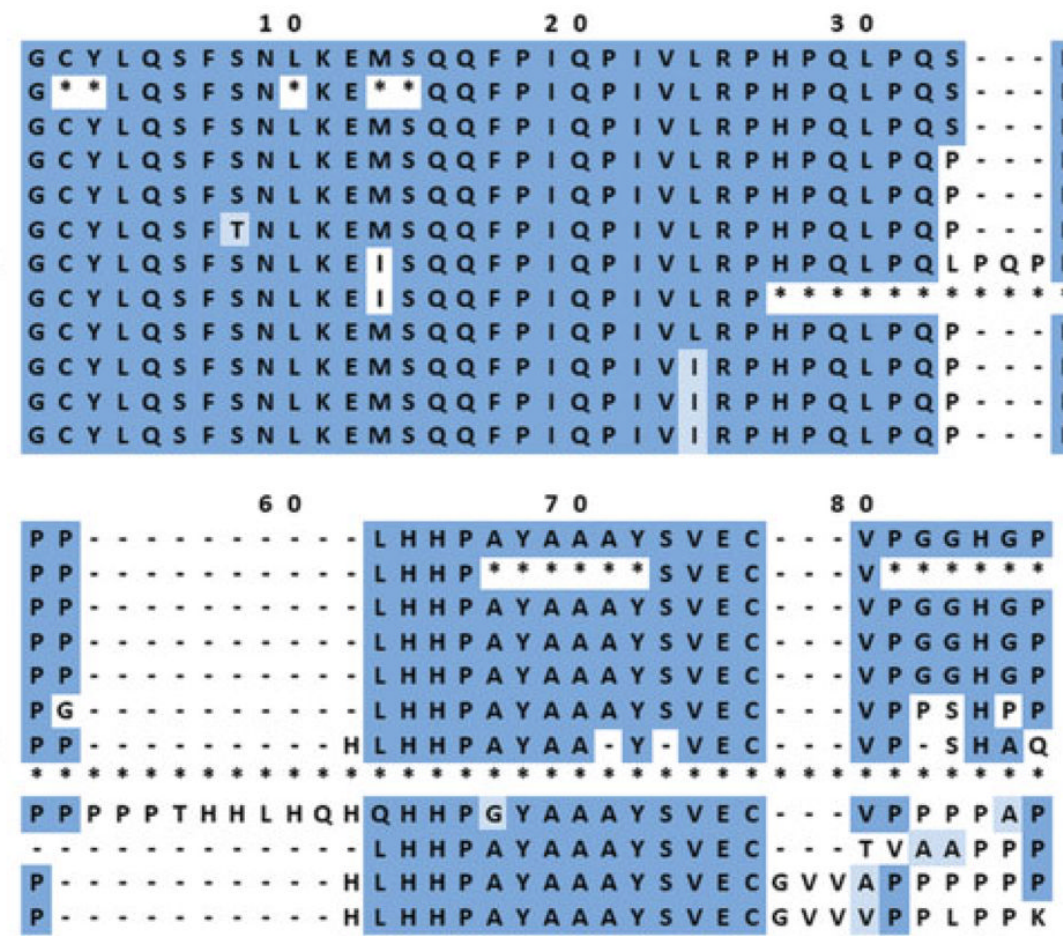

FIG. 4.

Conservation of region D in several Drosophila species. An alignment of region D using the twelve sequenced and annotated Drosophila genomes. The dark blue boxes denote areas of identity, the light blue boxes indicate areas of conservation and the white boxes indicate nonconservation. The dashed lines indicate true gaps in the sequence. The asterisks represent areas in which the sequences from NCBI and Flybase contradict and thus an assignment could not be reliably made. We divided the most distal section of the C-terminal tail (amino acids after region $\mathrm{C}$ ) into two halves. The first section (called region $\mathrm{D}$ ) appears to be required for the inhibitory role of the Optix C-terminal segment. Section E, in contrast, appears to be completely dispensable. 


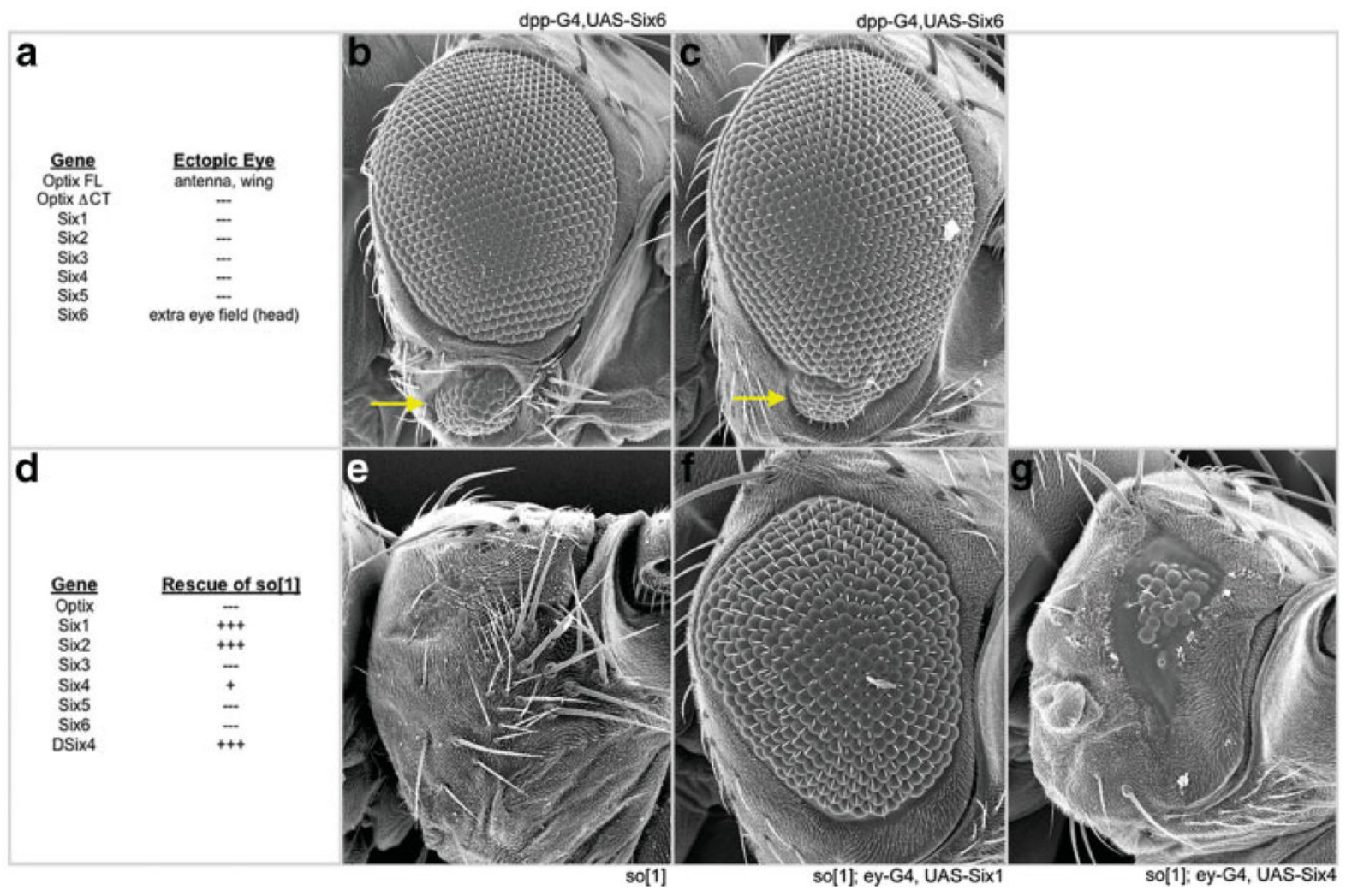

FIG. 5.

Induction of ectopic eyes and rescue of so[1] loss-of-function mutants. (a,d) Summary of ectopic eye and rescue experiments. (b,c and e-g) Scanning electron micrographs of adult compound eyes. The identity of individual UAS constructs is listed at the bottom right of each panel. All constructs were expressed in the eye using the ey-GAL4 driver. Arrows demarcate extra-eye fields. ( $(-)$ indicates no rescue. $(+)$ indicates weak rescue. $(+++)$ indicates significant rescue but not to fully wild type levels. Anterior is to the right. Note that ectopic eyes derived from expression of optix within the antenna and wing were described by Seimiya and Gehring (2000) and Weasner et al. (2007), respectively. [Color figure can be viewed in the online issue, which is available at www.interscience.wiley.com.] 